\title{
Real Time Nanogravimetric Monitoring of Corrosion for Nuclear Decommissioning
}

\author{
I. Tzagkaroulakis and C. Boxall \\ Engineering Department, Lancaster University, Lancaster, LA1 4YR, UK
}

Monitoring and understanding of corrosion on nuclear sites plays a key role in safe asset management (predicting plant life, assessing efficacy of corrosion inhibitors for plant lifetime extension) and supporting informed choice of decontamination methods for steels due for decommissioning. Recent advances in Quartz Crystal Nanobalance (QCN) technology offer a means to monitor corrosion in-situ in radiologically harsh environments, in real time and with high sensitivity. Oxalic acid has been widely used in nuclear plants and installations as a corrosion inhibitor for carbon steels and as a decontamination cleaning agent due to its ability to remove rust from the surface of ferritic metals and alloys. As an exemplar system for decontamination, the corrosion behavior of mild carbon steel and pure iron samples in $1 \mathrm{wt} \%$ to $8 \mathrm{wt} \%$ oxalic acid solutions have, for the first time, been measured and compared in real time and in situ using the QCN. Corrosion rates measured using the QCN are found to agree with those obtained using corrosion current ( $\mathrm{i}_{\text {CORR }}$ ) measurements, with the added advantages of: (i) real time and potentially in situ and higher sensitivity measurement; (ii) reduced uncertainty in the conversion of the QCN measured frequency change to a mass change-based corrosion rate compared to the conversion of the $i_{\text {corr }}$ measured by LSV with Tafel extrapolation to similar; (iii) the provision of mechanistic insights into the action of oxalic acid on Fe-rich steels.

\section{Introduction}

Metals are ubiquitous on nuclear sites as plant and construction materials. Monitoring and understanding metal corrosion plays a key role in safe asset management (predicting plant longevity, assessing efficacy of corrosion inhibitors for plant lifetime extension) and supporting informed choice of decontamination methods for steel structures due for decommissioning.

There are four common means of monitoring rates of corrosion, all with practical limitations: coupon testing (unsuitable for real time monitoring); electrical resistance monitoring (has sensitivity issues); linear polarisation resistance (LPR); and galvanic monitoring (both indirectly inferring corrosion rate in $\mathrm{mm} /$ year from current measurements).

Recent advances in Quartz Crystal Nanobalance technology (1) offer a means to avoid these issues. The QCN measures minute changes in frequency of a quartz crystal resonator with weight gain/loss. Using the Sauerbrey equation, the frequency change observed during corrosion testing can be converted to an instantaneous mass change (gain or loss, depending upon the direction of frequency change) and thus corrosion rate with nanogram sensitivity.

The recent availability of a wide range of metal coated crystal resonators (steel, $\mathrm{Al}, \mathrm{Fe}, \mathrm{Ti}$ etc) makes the QCN ideal for real time, instantaneous corrosion monitoring of steels. Its sensitivity makes it ideal for the measurement of low corrosion rates (e.g. passive corrosion) whilst its capacity for remote deployment allows for use in radiologically harsh environments with reduced operator exposure times, a key safety consideration for radiation workers. 
In this paper, we describe what we believe to be the first time use of the QCN for the in situ, real time monitoring corrosion. Particularly, we describe its development in the context of the monitoring of corrosion rates in an exemplar, non-radioactive surrogate system for the decontamination of storage tanks containing High Level (radioactive) Waste (HLW). The exemplar system that we have chosen is the corrosion behavior of mild carbon steel and pure iron samples in $1 \mathrm{wt} \%$ to $8 \mathrm{wt} \%$ oxalic acid solutions.

Oxalic acid has been used widely to decontaminate cooling circuit pipework in water cooled reactors, as an oxide deposit remover in power plants (2), as a decontamination agent during the Post Operational Clear Out (POCO) of nuclear facilities due for decommissioning. In particular, it is currently being studied as an Enhanced Chemical Cleaning (ECC) decontamination agent in the decommissioning of mild carbon steel-based, liquid HLW storage tanks at the Hanford and Savannah River Sites (SRS) in the US (3). In this, oxalic acid's duel properties as a rust remover and as a corrosion inhibitor for carbon steel surfaces $(4,5)$ are exploited - the former property making oxalic acid perfect for the treatment of highly contaminated surfaces where the contamination is entrained in the corrosion product layer, whilst the latter property protects the underlying fabric of the tank against corrosive dissolution, thus preserving its integrity and preventing the highly undesirable leaking of liquid HLW to the environment. Typically 1-8 wt\% oxalic acid is used for the ECC $(3,5,6)$.

However, the harsh chemical and radiological environments within the tanks (high temperature due to radioactive decay-derived self-heating, high oxidative stress due to the radiolysis of, inter alia, water) may compromise this protection. Additionally, given that the tanks are comprised of mild carbon steel, the liquid HLW stored within is rendered heavily alkaline ([hydroxide] $>1 \mathrm{~mol} \mathrm{dm}^{-3}$ ) to minimize corrosion of the tank fabric; the addition of significant quantities of oxalic acid may at least in part neutralize this alkaline protection.

Thus, in the interests of maintaining tank integrity, the potentially corrosive effect of any oxalic acid-based decontamination process on the mild carbon steel tank body must be understood and carefully monitored during process deployment. This requirement therefore affords an ideal opportunity for achieving the twin objectives of the development of the QCN as a corrosion monitoring device, whilst providing new insights into the behavior of a corrosion vulnerable system. Accordingly, we report here on the development and use, for the first time, of the QCN as a real time and in situ corrosion monitor in the context of a study of the corrosion behavior of mild carbon steel and pure iron samples (as tank wall surrogates) in $1 \mathrm{wt} \%$ to $8 \mathrm{wt} \%$ oxalic acid solutions (as ECC agent simulants).

\section{Experimental}

\section{Materials and Reagents}

All reagents were ACS reagent grade or higher and purchased from Fisher Scientific (Loughborough, UK) or Sigma Aldrich (Gillingham, Dorset, UK) and used without further purification. All solutions were prepared using doubly deionised water. Double deionised water was prepared using a Direct-Q 3 UV Millipore water purification system (Millipore, Watford, UK) to a resistivity of $18.2 \mathrm{M} \Omega . \mathrm{cm}$. Unless otherwise noted, all solutions were deaerated by purging with high purity $\mathrm{N}_{2}$ (BOC, UK) for 15 minutes before and during each electrochemical experiment.

\section{Fabrication of Mild Carbon Steel and Iron Disc Electrodes}

Mild carbon steel and iron disc electrodes were fabricated by mounting $10 \mathrm{~mm}$ diameter length of mild carbon steel rod (iron 98.81\% - 99.26\%, carbon 0.18\%, manganese 0.6 0.9\%, phosphorus 0.04\% max, sulphur 0.05\% max, Goodfellows, UK) or pure iron rod 
(99.99+\% purity, Goodfellows, UK) onto a brass head using silver loaded epoxy (ABL (STEVENS) Resin \& Glass, Cheshire, UK www.resin-supplies.co.uk). This assembly was then placed in a cylindrically shaped mould and sealed in epoxy resin with 6 days curing at $50^{\circ} \mathrm{C}$ (ABL (STEVENS) Resin \& Glass) in order to produce a suitable working electrode. The resultant $10 \mathrm{~mm}$ diameter disc electrodes were polished before each experiment using decreasing grades of SiC abrasive papers (Struers FEPA P\#1000, Buehler-Met P1200, Struers FEPA P\#2400), followed by decreasing grades of diamond slurries $(6,3,1 \mu \mathrm{m})$ (Marcon) and a final polish on a clean polishing pad soaked in distilled water. After polishing the electrodes were washed with deionized water to remove any debris remaining from the polishing stages.

\section{Electrochemical measurements}

All electrochemical measurements, unless stated otherwise, were carried out on a Gamry Model 600 Potentiostat/Galvanostat/ZRA (SciMed Ltd., Stockport, Cheshire, UK) using Gamry Resonator and EChem Analyst control, data acquisition and analysis software packages. All cyclic voltammetric (CV) and linear sweep voltammetry (LSV) experiments were conducted in a 3 electrode cell with a Pt wire mesh (Goodfellow Cambridge Ltd., Huntingdon, UK) counter electrode and a Ag/AgCl (Alvatek Ltd. Tetbury, Gloucestershire, UK) reference electrode used in a double junction (Alvatek Ltd. Tetbury, Gloucestershire, UK) configuration with saturated $\mathrm{K}_{2} \mathrm{SO}_{4}$ solution. For both $\mathrm{CV}$ and LSV experiments a sweep rate of $0.01 \mathrm{~V} / \mathrm{s}$ was used with a staircase step of $4.42 \mathrm{mV}$.

Open circuit potential $\left(\mathrm{E}_{\mathrm{OC}}\right)$ measurements were conducted using a simple 2 electrode cell in conjunction with the same potentiostat and $\mathrm{Ag} / \mathrm{AgCl}$ reference electrode as above.

\section{Electrochemical Quartz Crystal Nanobalance Experiments}

The electrochemical quartz crystal nanobalance (EQCN) is a well-established method for the measurement of small changes in electrode mass due to reactions occurring at the electrodesolution interface. A detailed description of QCN theory may be found in various texts (710). Assuming mass is rigidly bound; the measured shift in the resonant frequency is converted to a mass change via Equation 1

$$
\Delta f=-\mathrm{C}_{f} \Delta m
$$

Where $\Delta f$ is the change in resonant frequency $(\mathrm{Hz}), \Delta m$ is the mass change $(\mathrm{g})$ and $C_{f}$ is the sensitivity constant. Using the Sauerbrey equation (11) the value of $\mathrm{C}_{f}$ can be determined from the resonant frequency and electroactive area of the crystals and is found to be 0.226 $\mathrm{Hz}\left(\mathrm{ng} \mathrm{cm}{ }^{-2}\right.$ ) for the $10 \mathrm{MHz}$ crystals employed here (vide infra). EQCN experiments were conducted using the above described Gamry Potentiostat model 600, coupled with a Gamry EQCN model 10M (SciMed Ltd.) allowing simultaneous QCN and Eod/potentiometric measurements to be taken. Experiments were performed in a ground floor lab sited directly on building foundations and isolated from heavy machinery and vibration sources.

\section{Preparation of Iron Crystals for Electrochemical QCN Experiments}

AT cut iron QCN piezoelectrodes with a $10 \mathrm{MHz}$ resonant frequency (International Crystal MFG, USA) were comprised of a $1.37 \mathrm{~cm}$ diameter Au blank (reverse side) electrode and a $0.51 \mathrm{~cm}$ diameter Fe working (forward side) electrode. These consisted of $100 \mathrm{~nm} \mathrm{Au}$ and Fe layers respectively, both over a $10 \mathrm{~nm}$ Ti binding layer. Piezoelectrodes were cleaned prior each experiment using a procedure described by Wilbraham et al. (11). Specifically, crystals were washed using a polypropylene eye dropper with, successively, chloroform, acetone and ethanol. The crystals were dried after each wash by nitrogen blowing. 


\section{Results and Discussion}

Comparing the Corrosion Behavior of Iron and Mild Carbon Steel in Oxalic Acid

Figure 1 shows the cyclic voltammetric behavior of iron and mild carbon steel disc electrodes scanned between $-1 \mathrm{~V}$ and $+1 \mathrm{~V}$ in 1 and $8 \mathrm{wt} \%$ oxalic acid solutions. For all figures, hydrogen evolution can be seen in the range $-1 \mathrm{~V}$ to $-0.5 \mathrm{~V}$. Each voltammogram also has the following features in common:

- During the forward going sweep: two oxidation peaks with onsets of $-0.5 \mathrm{~V}$ and $+0.35 \mathrm{~V}$, the former exhibiting an abrupt current decrease at $\sim-0.1 \mathrm{~V}$ in $1 \%$ oxalic acid and $\sim-0.2 \mathrm{~V}$ in $8 \%$ oxalic.

- During the reverse going sweep: one sharp oxidation peak with a reverse going onset / peak potential of $+0.2 \mathrm{~V}$, dropping away to zero current by $0 \mathrm{~V}$.

These features can be interpreted with the assistance of the simplified $\mathrm{E}_{\mathrm{h}} \mathrm{pH}$ diagrams for the Fe-oxalate- $\mathrm{H}_{2} \mathrm{O}$ and $\mathrm{Fe}-\mathrm{H}_{2} \mathrm{O}$ systems shown in Figures 2A and 2B and adapted from the work of Pourbaix (12) and Saltykov et al. (13) respectively.

From both Figures $2 \mathrm{a}$ and $2 \mathrm{~b}$, and the fact that the $\mathrm{pHs}$ of $1 \%$ and $8 \%$ oxalic acid solutions are 1.3 and 0.85 respectively, it can be see that the onset of the first wave in the forward going sweep of all CVs in Figure 1 is due to the oxidation of $\mathrm{Fe}$ metal to $\mathrm{Fe}^{2+}$ ions. In the absence of oxalate, Figure $2 \mathrm{a}$ indicates that this reaction would be expected to continue unimpeded up to $\sim+0.4 \mathrm{~V}$ vs. $\mathrm{Ag} / \mathrm{AgCl}$ at which point further oxidation to $\mathrm{Fe}^{3+}$ occurs with consequent formation of solid phase $\mathrm{Fe}_{2} \mathrm{O}_{3}$, most likely in the form of $\gamma-\mathrm{Fe}_{2} \mathrm{O}_{3}$, maghemite (proved by recording the photocurrent of the polarized electrode (14)). However, in the presence of oxalate, Figure $2 \mathrm{~b}$ indicates that $\mathrm{Fe}^{2+}$ generated at $\mathrm{E}>-0.5 \mathrm{~V}$ may, as a result of locally exceeding the solubility product, precipitate as $\mathrm{FeC}_{2} \mathrm{O}_{4}$ at the electrode surface.
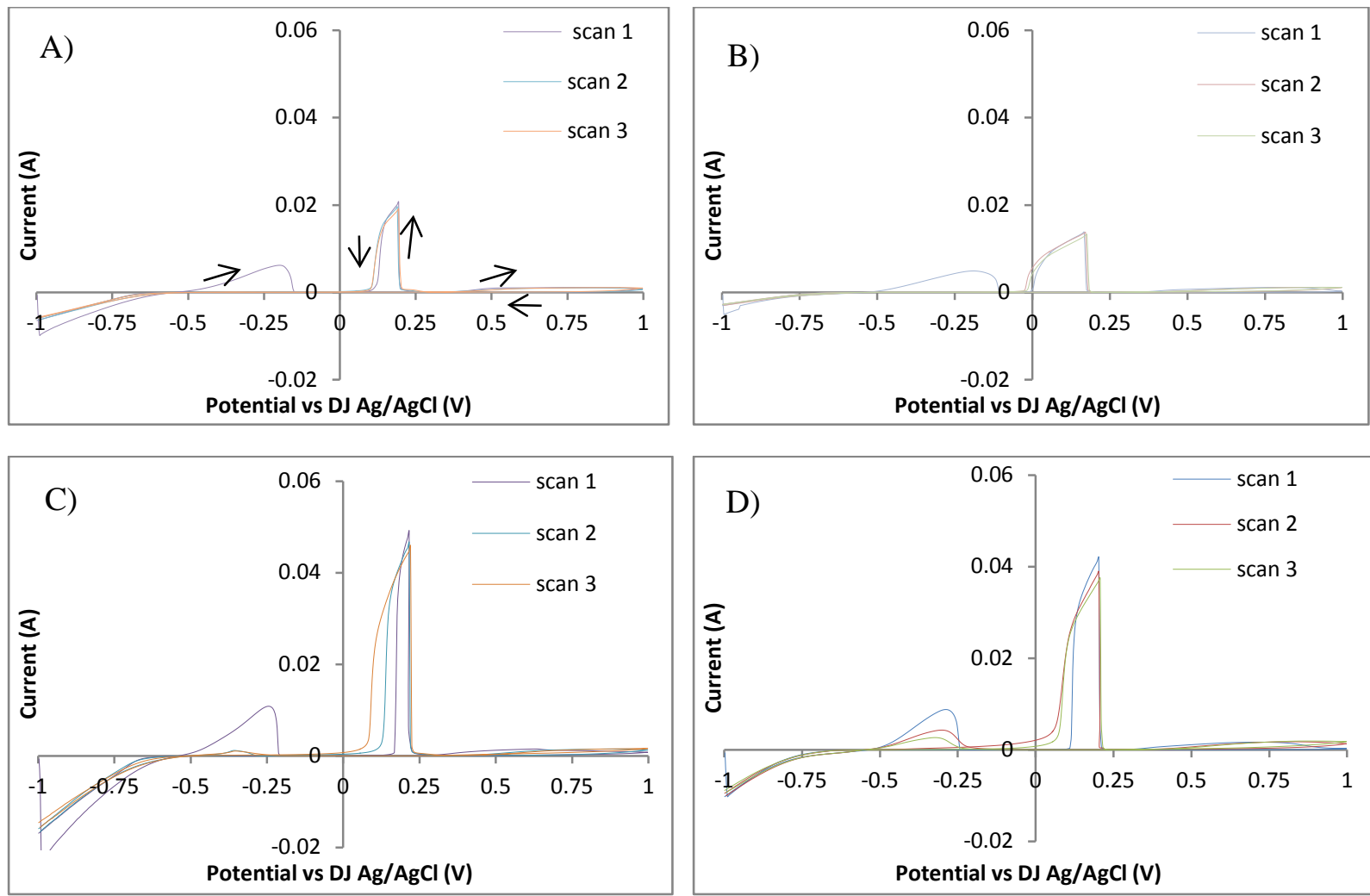

Figure 1. Comparing cyclic voltammograms of mild carbon steel, (A) and (C), and iron electrodes, (B) and (C), in 1wt \% oxalic acid, $\mathrm{pH}=1.3$ (A) and (B) and 8wt\% oxalic acid, $\mathrm{pH}=0.85(\mathrm{C})$ and $(\mathrm{D})$. 

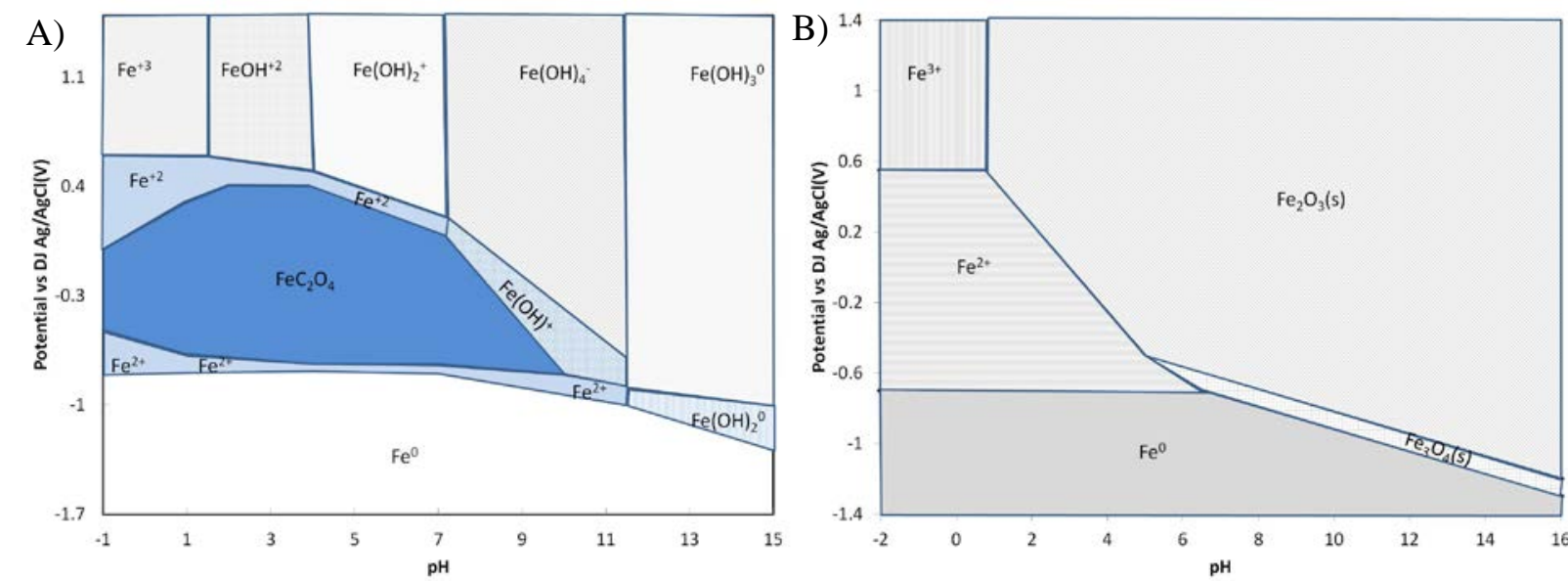

Figure 2. Simplified $\mathrm{E}_{\mathrm{h}}-\mathrm{pH}$ (Pourbaix) diagrams for the (A) Fe-oxalate- $\mathrm{H}_{2} \mathrm{O}$ and (B) $\mathrm{Fe}-\mathrm{H}_{2} \mathrm{O}$ systems, adapted from the work of Saltykov et al. (13) and Pourbaix (12).

That we see an abrupt current decrease associated with this peak at $\mathrm{E}>\sim-0.2 \mathrm{~V}$ suggests that this ferrous oxalate phase passivates the electrode surface.

This passive behavior maintains in all CVs of Figure 1 until $\mathrm{E} \sim+0.35 \mathrm{~V}$ at which point an oxidation current begins to flow. From Figure 2A, this can be seen to be due to the oxidation of $\mathrm{FeC}_{2} \mathrm{O}_{4}$ to form free solution oxalate and $\mathrm{Fe}^{3+}$. However, as discussed above, Figure 2B indicates that at these pHs, so-released $\mathrm{Fe}^{3+}$ will precipitate as $\mathrm{Fe}_{2} \mathrm{O}_{3}$ at the electrode surface, so inducing a secondary electrode passivation.

Thus, during the return sweep, the electrode surface is initially comprised of a layer of $\mathrm{Fe}_{2} \mathrm{O}_{3}$. However, Figure 2B indicates that at $\mathrm{E}<\sim 0.4 \mathrm{~V}$, this $\mathrm{Fe}_{2} \mathrm{O}_{3}$ layer is reduced back to solution phase $\mathrm{Fe}^{2+}$, exposing the $\mathrm{Fe}$ metal surface beneath. Almost counter-intuitively, this $\mathrm{Fe}_{2} \mathrm{O}_{3}$ reduction manifests itself as the sharp oxidation peak seen in all of the reverse scans of Figure 1; however, this can be understood by realizing that, at the potential of $\mathrm{Fe}_{2} \mathrm{O}_{3}$ reduction, the exposed Fe metal will be subject to an oxidative dissolution reaction, giving rise to the sharply rising oxidation current observed. The attenuation of that current to zero in all CVs of Figure 1 can then be easily understood in the context of Figure 2A: simply, the $\mathrm{Fe}^{2+}$ released by the reduction of the $\mathrm{Fe}_{2} \mathrm{O}_{3}$ surface layer and the oxidation of the underlying Fe metal again interacts with solution oxalate to generate a layer of passivating $\mathrm{FeC}_{2} \mathrm{O}_{4}$ at the electrode surface.

Finally it appears that, once generated at the electrode surface during the reverse sweep, this layer of passivating $\mathrm{FeC}_{2} \mathrm{O}_{4}$ retains into the forward going sweep of the next cycle. This is indicated by the height of the first peak in the forward going sweep decreasing with increasing scan number - and suggests that $\mathrm{FeC}_{2} \mathrm{O}_{4}$, once formed, is kinetically resistant to reduction back to Fe metal.

It is apparent from these results that mild carbon steel and pure iron behave in a broadly similar fashion with respect to their voltammetric behaviour in the presence of oxalic acid. This similarity is underscored by the results of Figure 3 which shows $E_{\mathrm{OC}}$ measurements for both electrode materials as a function of oxalic acid concentration in the range 1 to $8 \mathrm{wt} \%$.

In the range 0 to $1 \mathrm{wt} \%$ oxalic acid, the measured $\mathrm{E}_{\mathrm{OC}}$ value abruptly decreases to $-0.49 \mathrm{~V}$ for both iron and mild carbon steel. It is then near-invariant with increasing oxalic acid concentration reaching values of $-0.49 \mathrm{~V}$ and to $-0.53 \mathrm{~V}$ for mild carbon steel and iron sample respectively. From these results, it is again apparent that mild carbon steel and pure iron behave in a broadly similar fashion with respect to their electrochemical behavior in the presence of oxalic acid - suggesting that we may use pure iron as a low carbon steel 


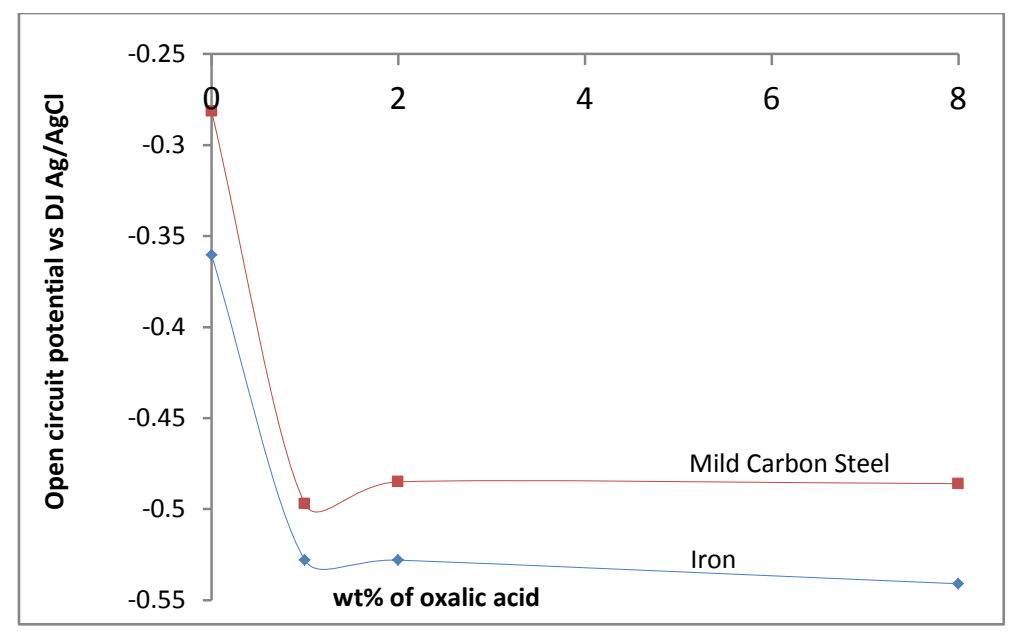

Figure 3. $E_{\mathrm{OC}}$ measurements for iron and mild carbon steel electrodes as a function of oxalic acid concentration in the range 1 to $8 \mathrm{wt} \%$.

surrogate, so allowing the use of less resource intensive iron-based QCN crystals (compared to high unit price steel crystals) for the monitoring of corrosion during the application of oxalic acid. As these crystals will be used destructively, this is an economically significant finding given that intended use of this technology is the monitoring of corrosion processes during the radioactive decontamination applications. Consequently, the QCN-based corrosion monitoring studies of the next section will focus on iron coated piezoelectrodes as mild steel surrogates.
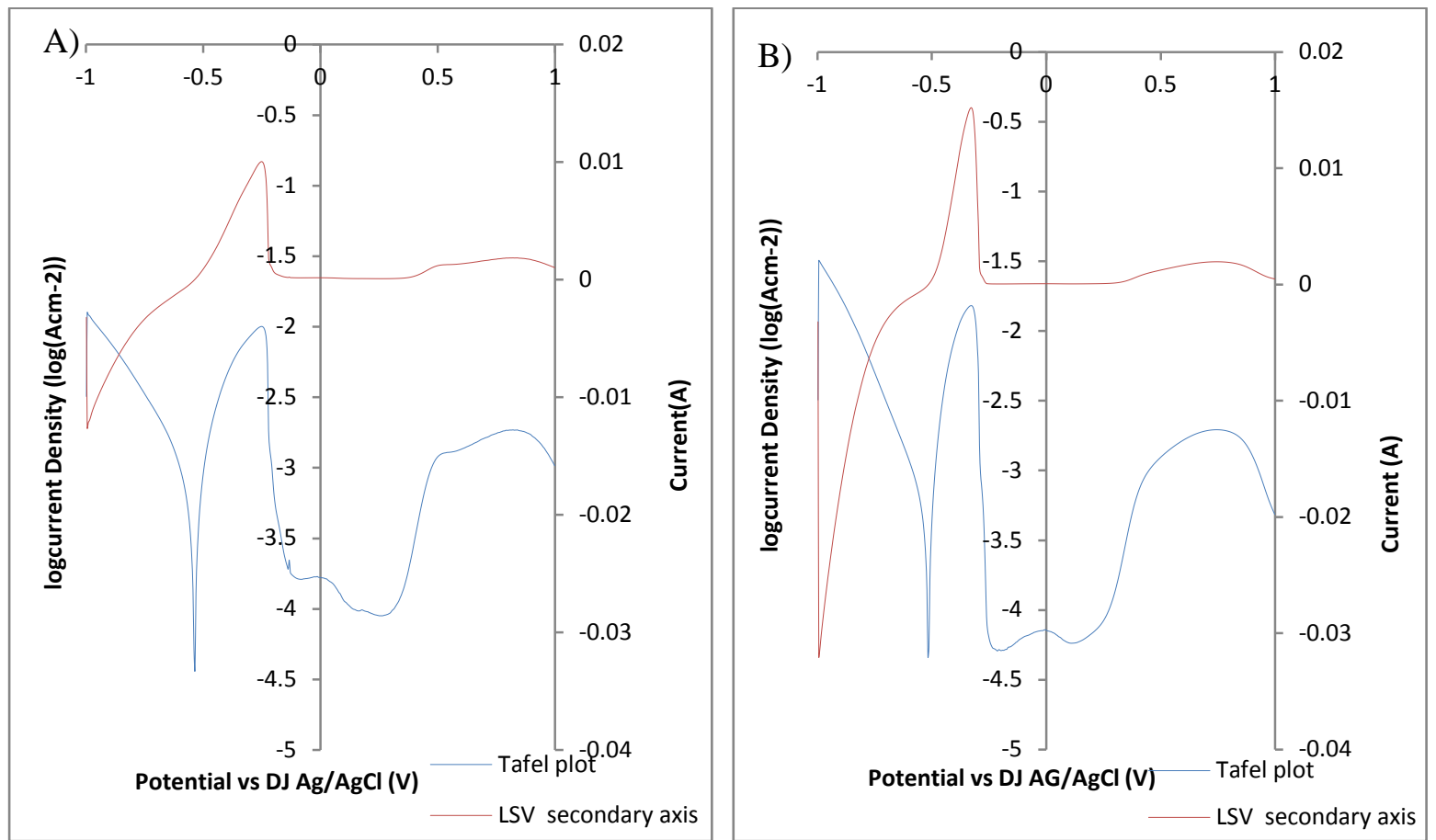

Figure 4. Forward going sweeps in the first scan cyclic voltammograms, and associated LSVs, of iron piezoelectrodes in (A) 1 and (B) $8 \mathrm{wt} \%$ oxalic acid. 

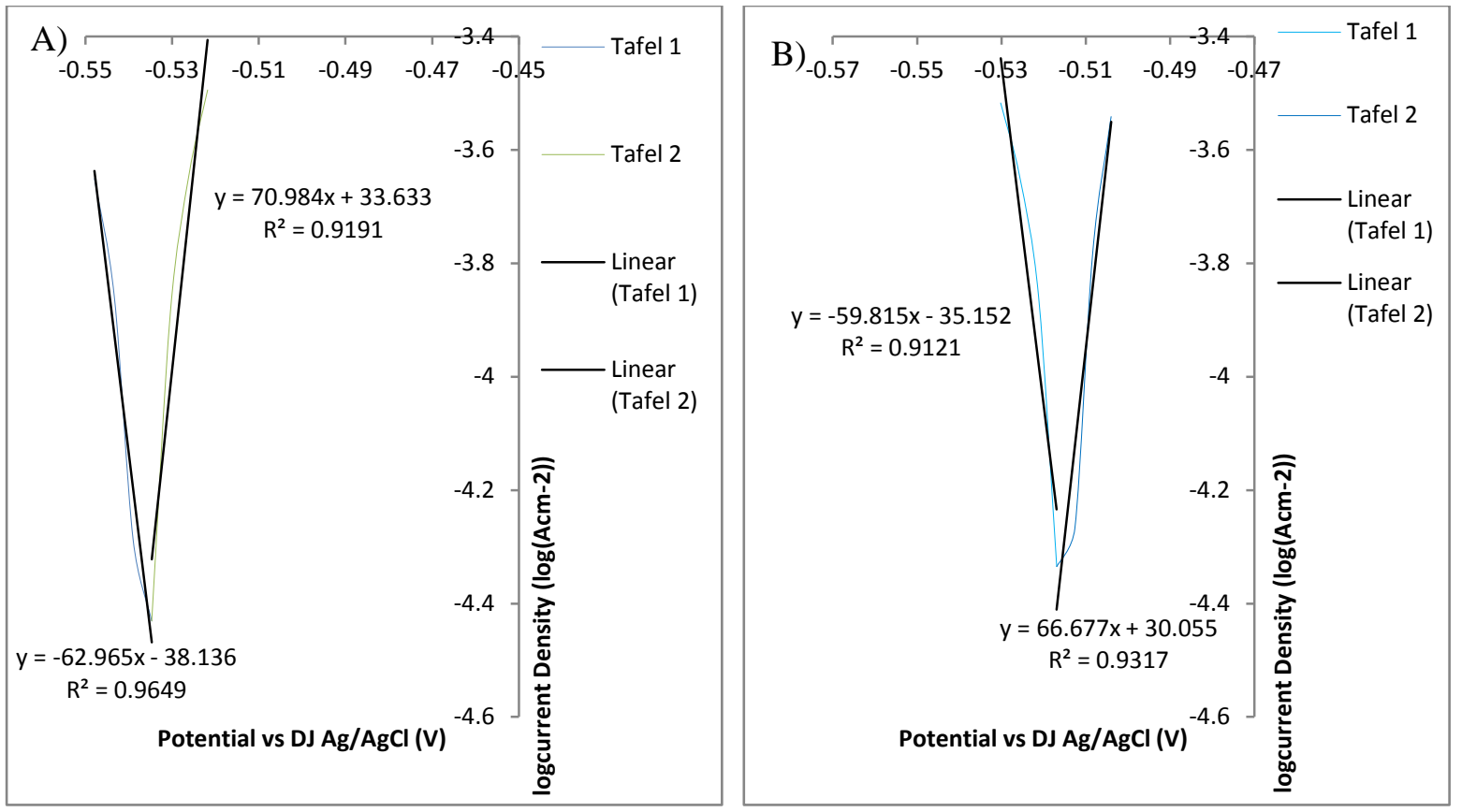

Figure 5. Tafel plots for data in the vicinity of the $\mathrm{E}_{\mathrm{CORR}}$, extracted from Figures $4 \mathrm{~A}$ and $4 \mathrm{~B}$ respectively. Extrapolated $\mathrm{E}_{\mathrm{CORR}}$ and $\mathrm{i}_{\mathrm{CORR}}$ values are given in Table 1.

Measuring the Corrosion Rate of Iron as a Mild Carbon Steel Surrogate with the EQCN Figures $4 \mathrm{~A}$ and $4 \mathrm{~B}$ show the forward going sweeps in the first scan cyclic voltammograms of iron piezoelectrodes in 1 and $8 \mathrm{wt} \%$ oxalic acid respectively. Figures $4 \mathrm{~A}$ and $4 \mathrm{~B}$ also show the corresponding LSVs, from which $E_{\text {CORR }}$ values for both systems may be seen to be $\sim-0.5 \mathrm{~V}$. The corrosion rate for each system may be obtained by application of $\mathrm{i}_{\mathrm{CORR}}$ as defined by the ASTM (18). Calculated from the data of Figure 4, Figure 5 shows the Tafel plots and

Table 1. Calculating the mass change using $i_{\text {CORR }}$ values obtained from the data of Figure 4 via the extrapolation of the Tafel relationships shown in Figure 5.

\begin{tabular}{ccc}
\hline Parameters & 1 wt\% oxalic acid & 8 wt\% oxalic acid \\
$E_{C O R R}(V)$ & -0.536 & -0.515 \\
$i_{C O R R}\left(\frac{\mu A}{\mathrm{~cm}^{2}}\right)$ & 38.5 & 48.2 \\
$K_{2}\left(g \mathrm{~cm}^{2} / \mu A m^{2} d\right)$ & $8.954 \times 10^{-3}$ & $8.954 \times 10^{-3}$ \\
$W$ & 55.845 & 55.845 \\
$\mathrm{n}$ & 2 & 2 \\
$E W=\frac{W}{n}$ & 27.92 & $\pm 1.2 \times 10^{-3}$ \\
Mass change rate $=K_{2} i_{C O R R} E W\left(\frac{g}{\mathrm{~cm}^{2} d}\right)$ & $\pm 0.96 \times 10^{-3}$ & \\
\hline
\end{tabular}


Tafel relationships for the anodic and cathodic current branches either side the $\mathrm{E}_{\mathrm{CORR}}$ for $\mathrm{Fe}$ piezoelectrodes immersed in 1 and $8 \mathrm{wt} \%$ oxalic acid. Extrapolated $\mathrm{E}_{\mathrm{CORR}}$ and $\mathrm{i}_{\mathrm{CORR}}$ values, obtained by equating the anodic and cathodic current branch Tafel relationships at each oxalic acid concentration, are given in Table 1 . In each case, the $\mathrm{i}_{\mathrm{CORR}}$ value can be converted to a mass change rate by use of Equation 2 (15):

$$
\text { Mass change rate }=K_{2} i_{C O R R} E W
$$

where $\mathrm{K}_{2}$ is a constant and EW is the equivalent weight, calculated assuming that the dominant mass change reaction is $\mathrm{Fe}$ metal to $\mathrm{Fe}^{2+}$. Mass change rates calculated using Equation 2 are also given in Table 1 and summarized in Table 2.
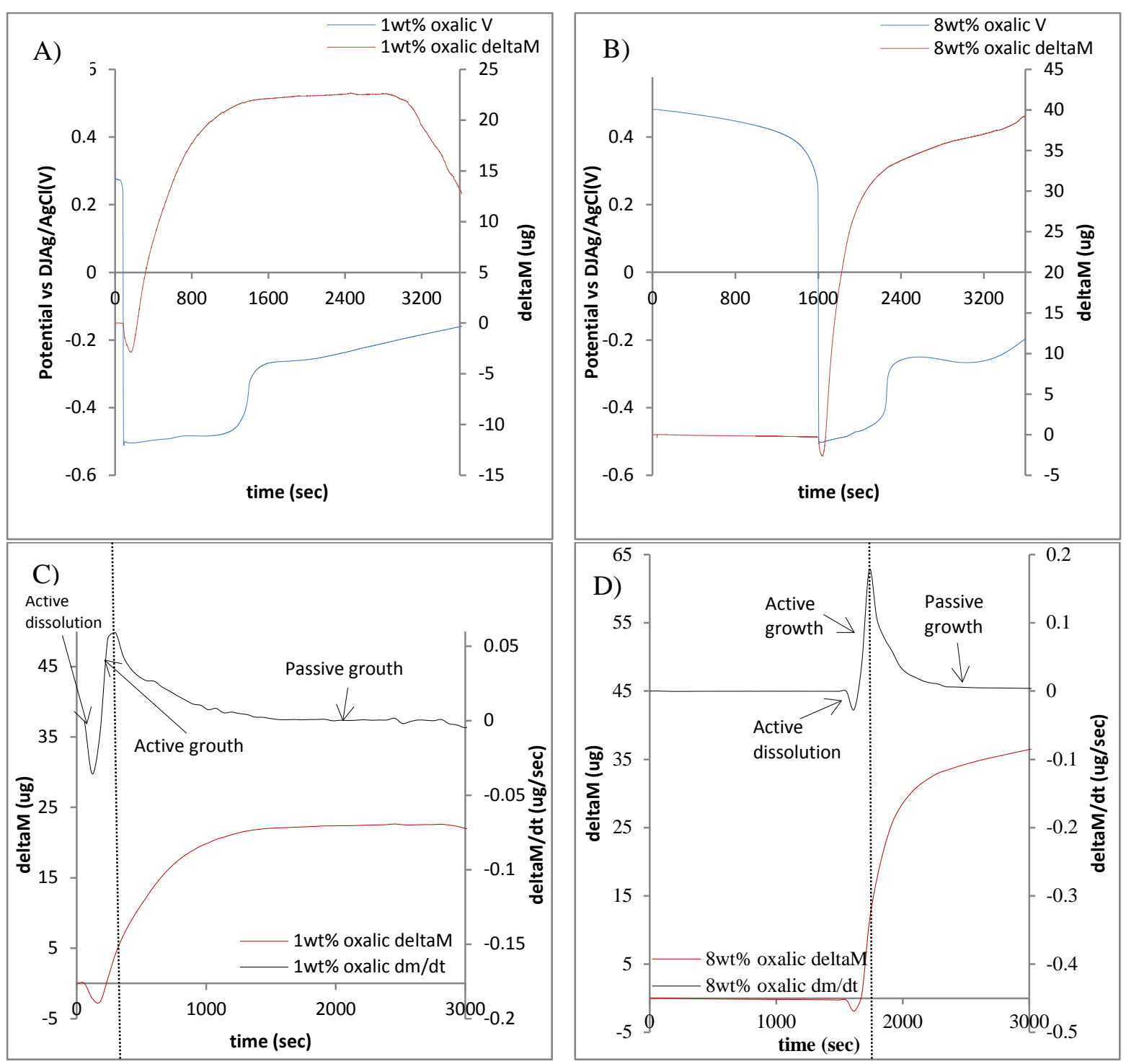

Figure 6. (A) \& (B): $E_{O C}$ and nanogravimetric data as a function of time, recorded from $\mathrm{Fe}$ piezoelectrodes using the EQCN in 1 and 8 wt\% oxalic acid respectively. Time $t=0$ corresponds to the point at which the QCN mounted Fe piezoelectrode is first immersed in the relevant oxalic acid solution. (C) \& (D): Derivatives with respect to time of the nanogravimetric data of Figures 6A and 6B respectively. For reader convenience, the source nanogravimetric data is also reproduced from Figures $6 \mathrm{~A}$ and $6 \mathrm{~B}$. 
Table 2. Comparing the mass change rates obtained using iCORR and Quartz Crystal Nanobalance measurements as a function of oxalic acid concentration.

\begin{tabular}{|c|c|c|c|}
\hline $\begin{array}{l}\text { oxalic acid } \\
\text { concentration } \\
\text { (wt } \%)\end{array}$ & $\begin{array}{c}\text { corrosion rate } \\
\text { obtained from } \\
\mathbf{i}_{\text {CORR }}\left(\mathrm{g} \mathrm{d}^{-1} \mathbf{c m}^{-2}\right)\end{array}$ & $\begin{array}{l}\text { maximum corrosion } \\
\text { rate obtained from } \\
\text { QCN }\left(\mathrm{g} \mathrm{d}^{-1} \mathrm{~cm}^{-2}\right)\end{array}$ & $\begin{array}{l}\text { passive corrosion } \\
\text { rate obtained from } \\
\text { QCN }\left(\mathrm{g} \mathrm{d}^{-1} \mathbf{c m}^{-2}\right)\end{array}$ \\
\hline 1 & $\pm 0.96 \times 10^{-3}$ & $+24.9 \times 10^{-3}$ & $+1.19 \times 10^{-3}$ \\
\hline 8 & $\pm 1.2 \times 10^{-3}$ & $+75.2 \times 10^{-3}$ & $+1.71 \times 10^{-3}$ \\
\hline
\end{tabular}

Figures $6 \mathrm{~A}$ and $6 \mathrm{~B}$ show $\mathrm{E}_{\mathrm{OC}}$ and nanogravimetric data as a function of time, recorded from Fe piezoelectrodes using the EQCN in 1 and $8 \mathrm{wt} \%$ oxalic acid. Time $\mathrm{t}=0$ corresponds to the point at which the QCN mounted Fe piezoelectrode is first immersed in the relevant oxalic acid solution. The $E_{\mathrm{OC}}$ and nanogravimetric traces at 1 and $8 \mathrm{wt} \%$ oxalic acid both exhibit 4 distinct sequential regions.

- Region 1: Little to no change in mass with an initial $\mathrm{E}_{\mathrm{OC}}$ of $+0.3 \mathrm{~V}$ in $1 \mathrm{wt} \%$ oxalic and $+0.5 \mathrm{~V}$ in $8 \mathrm{wt} \%$.

- Region 2: A rapid decrease in the $\mathrm{E}_{\mathrm{OC}}$ to $\sim-0.5 \mathrm{~V}$ for both oxalic acid concentrations accompanied by small decrease in piezoelectrode mass.

- Region 3: A rapid increase in mass accompanied by a gradual increase in $\mathrm{E}_{\mathrm{OC}}$ from $-0.5 \mathrm{~V}$ to $\sim-0.4 \mathrm{~V}$. The end of region 3 is marked by an abrupt increase in $\mathrm{E}_{\mathrm{OC}}$ to $\sim-0.25 \mathrm{~V}$ and an almost equally abrupt slowing of the rate of mass increase.

- Region 4: A near constant $\mathrm{E}_{\mathrm{OC}}$ of $-0.25 \mathrm{~V}$ at both oxalic acid concentrations, accompanied by an increase in piezoelectrode mass, albeit at a much slower rate than in region 3.

The $\mathrm{E}_{\mathrm{OC}}$ behaviour in regions 1 and 2 is similar to that seen by Cartledge when stainless steel 347 electrodes are immersed in $\mathrm{pH} 1.46$ sulfuric acid (16), the region 1 behavior being attributed to the slow acid dissolution of a layer of $\mathrm{Fe}_{2} \mathrm{O}_{3}$ generated at the electrode surface by aerial oxidation prior to electrode immersion $(16,17)$. Once this layer of $\mathrm{Fe}_{2} \mathrm{O}_{3}$ has thinned to the point that the underlying Fe metal is exposed, reduction of the remaining $\mathrm{Fe}_{2} \mathrm{O}_{3}$ to $\mathrm{Fe}^{2+}$ galvanically couples with the oxidation of $\mathrm{Fe}$ metal to $\mathrm{Fe}^{2+}$, resulting in an abrupt decrease in $\mathrm{E}_{\mathrm{OC}}$ and an instantaneous mass loss at the electrode surface $(16,17)$, as seen in region 2.

As in the CVs of Figures 1 and 4, this efflux of $\mathrm{Fe}^{2+}$ to solution then interacts with solution oxalate to generate a layer of passivating $\mathrm{FeC}_{2} \mathrm{O}_{4}$ at the electrode surface with a corresponding rapid increase in piezoelectrode mass and an arrest in the downward / negative trajectory of the $\mathrm{E}_{\mathrm{OC}}$, region 3. Finally, both $\mathrm{Fe}$ metal oxidation and consequent ferrous oxalate formation is self-limited by the presence of the $\mathrm{FeC}_{2} \mathrm{O}_{4}$ layer at the electrode surface and the $\mathrm{E}_{\mathrm{OC}}$ comes to rest at a potential that, as can be seen from Figures 2 and 4, corresponds to a region of $\mathrm{FeC}_{2} \mathrm{O}_{4}$ derived electrode passivity i.e. the $\mathrm{FeC}_{2} \mathrm{O}_{4}$ corrosion product layer has entered a region of passive growth.

Having explained the forms of the $\mathrm{E}_{\mathrm{OC}}$ and mass vs. time traces of Figure $6 \mathrm{~A}$ and $6 \mathrm{~B}$, it is informative to consider the first differentials of the mass $v s$. time traces in order to obtain corrosion rates, in the form of mass change rates, as a function of time. The resultant $(\mathrm{dm} / \mathrm{dt})$ vs. time plots are shown in Figures 6C and 6D.

Of particular interest is the behavior of these $(d m / d t)$ vs. time plots in regions of Figures $6 \mathrm{~A}$ and $6 \mathrm{~B}$ where substantive mass changes are occurring i.e. regions 3 and 4 . Here it can be seen that the $(d m / d t)$ vs. time traces rise to a maximum corrosion rate in region 3 before relaxing back to a smaller, constant corrosion rate in region 4. Again the general form of these traces are consistent with there being an initial rapid growth of a $\mathrm{FeC}_{2} \mathrm{O}_{4}$ layer in region 3 that then self-limits, undergoing slower passive growth in region 4.

Table 2 reports both the QCN determined maximum observed corrosion rate of region 3 as well as the lower, constant corrosion rate of region 4 for both investigated concentrations of 
oxalic acid. It also compares these with the corrosion rates determined from the $\mathrm{i}_{\text {CORR }}$ measurements and calculations of Figures 5A and 5B and Table 1. Table $1 \& 2$ and Figure 6 allow for a number of observations to be made.

1. There is very good agreement between the respective corrosion rates calculated from $\mathrm{i}_{\text {CORR }}$ values and those calculated from QCN data recorded in the passive growth region (Table 2 ), indicating that $\mathrm{i}_{\mathrm{CORR}}$ derived rates reflect passive growth rates as well.

2. The QCN allows for time dependence of corrosion rates to be measured in situ and in real time (Figure 6); $i_{\text {CORR }}$ based measurements allow for neither of these.

3. Corrosion rates calculated using QCN data explicitly describe whether the associated mass changes correspond to mass increases or mass losses (Figure 6); no such information is available from corrosion rates calculated using $\mathrm{i}_{\mathrm{CORR}}$ values.

4. Corrosion rates calculated using $\mathrm{i}_{\mathrm{CORR}}$ data require that assumptions are made as to the half reactions that are galvanically coupling in the overall corrosion process. For example, the corrosion rate calculation of Table 1 explicitly assumes, through the EW value, that the anodic half reaction is the oxidation of Fe metal to solution phase $\mathrm{Fe}^{2+}$.

5. In contrast, corrosion rates calculated using QCN data require no assumptions be made regarding the galvanically coupling half-reactions; indeed the coupling of nanogravimetric data with $\mathrm{E}_{\mathrm{OC}}$ measurements afforded by the EQCN can allow for the explicit identification of the reactions giving rise to the measured corrosion derived mass change. For example Figures 6A and 6B, by comparison with Figures 2 and 4, reveal that the mass increases measured in each case are due to the oxidation of $\mathrm{Fe}$ metal to $\mathrm{Fe}^{2+}$ followed by the deposition of a $\mathrm{FeC}_{2} \mathrm{O}_{4}$ corrosion product at the electrode surface rather than the $\mathrm{Fe} / \mathrm{Fe}^{2+}$ oxidation alone, as assumed in the $\mathrm{i}_{\mathrm{CORR}}$ based corrosion rate calculation.

Indeed, if the EW value used in Table 1 to calculate the mass change-based corrosion rate from the $i_{\text {CORR }}$ data is changed to reflect ferrous oxalate deposition $(E W=44)$ rather than ferrous ion discharge to solution $(\mathrm{EW}=27.92)$, the respective $\mathrm{i}_{\mathrm{CORR}}$-derived corrosion rates for the 1 and $8 \mathrm{wt} \%$ oxalic acid can be recalculated to be $1.51 \times 10^{-3}$ and $1.89 \times 10^{-3} \mathrm{~g} \mathrm{~d}^{-1} \mathrm{~cm}^{-2}$. These values are in even better agreement with those calculated from QCN data in the passive region, again underlining the accuracy of the EQCN as a corrosion monitoring device.

\section{Conclusions}

The electrochemical quartz crystal nanobalance has been used as a corrosion monitor for steel analogues for the first time. Using the iron / oxalic acid system as a low resource-intensive surrogate for the mild steel / oxalic acid system under conditions relevant to nuclear decommissioning, the corrosion behavior of iron samples in $1 \mathrm{wt} \%$ to $8 \mathrm{wt} \%$ oxalic acid solutions have, for the first time, been measured and compared in real time and in situ using the QCN.

Corrosion rates measured using the QCN are found to agree with those obtained using corrosion current ( $\left.\mathrm{i}_{\mathrm{CORR}}\right)$ measurements, with the added advantages of:

(i) Instantaneous and real time measurement;

(ii) potentially in situ and higher sensitivity measurement;

(iii) reduced uncertainty in the conversion of the QCN measured frequency change to a mass change-based corrosion rate compared to the conversion of the LSV-determined $\mathrm{i}_{\text {corr }}$ to similar;

(iv) the provision of mechanistic insights into the action of oxalic acid on Fe-rich steels.

The use of the EQCN to study the corrosion of a range of steels under simulated radiological conditions relevant to nuclear decontamination and decommissioning are currently ongoing. 


\section{Acknowledgments}

We thank the UK Nuclear Decommissioning Authority (NDA/NNL Agreement No 1010350) and the Lloyd's Register Foundation for financial support. Lloyd's Register Foundation supports the advancement of engineering-related education, and funds research and development that enhances safety of life at sea, on land and in the air.

\section{References}

1. P. S. Foran and C. Boxall, in Mater. Res. Soc. Symp. Proc., 1406 (2012).

2. R.M. Sellers and W.J. Williams Faraday Discuss Chem. Soc., 77, 265-274, (1984).

3. R. H. Spires, E. Ketusky, B. Barton and D. Jones, WM 2010, Mar. 7-11, Phoenix, AZ, (2010).

4. S. Rahfield and B. Newman, Boyle-Midway Household Products, Patent US 4828743 A (1989).

5. M. A. Streicher, Du Pont de Nemours and Company, Patent US 2793190 A (1957).

6. E. Ketusky, K. Subramanian and B. Wiersma - 11413' WM2011, Phoenix, AZ, (2011).

7. A. J. Bard, R. Parsons, J. Jordan, Standard Potentials in Aqueous Solution, CRC Press, (1985).

8. D.A. Buttry, Electroanal. Chem., 17, 1 - 85 (1991).

9. D.A. Buttry, Electrochemical Interfaces: Modern Techniques for In-Situ Interface Characterisation, H.D. Abruna, Editor, VHC Publishers Inc., 529-566, (1991).

10. M.R. Deakin, D.A. Buttry, Anal. Chem., 61, 1147A-1154A, (2008).

11. R.J. Wilbraham, C. Boxall and R. J. Taylor, J. of Photochemistry and Photobiology A: Chemistry, 249, 21 (2012).

12. M. Pourbaix, Atlas of Electrochemical Equilibria on Aqueous Solutions $2^{\text {nd }}$ Edition, NACE, Houston, Texas, USA (1974).

13. S. N. Saltykov, G. V. Makarov, E. L. Toroptseva, and Ya. B. Filatova, Protection of Metals, 40, No. 1, 56 (2004).

14. J.L. Camalet, J.C. Lacroix, S. Aeiyach, K.C. Ching and P.C. Lacaze, Synthetic Metals, 93, 133 (1998).

15. ASTM-Standards, Standard Practice for Calculation of Corrosion Rates and Related Information from Electrochemical Measurements, Designation: G 102 - 89 (1999).

16. G.H. Cartledge Journal of the Electrochemical Society, 104, 420 (1957).

17. Z. Qin, B. Demko, J.J. Noël, D.W. Shoesmith, F. King, R. Worthingham, and K. Keith Corrosion 2004, 60, 906 (2004). 